GLASNIK MATEMATIČKI

Vol. 40(60)(2005), $303-312$

\title{
DIRECT LIMIT OF MATRIX ORDERED SPACES
}

\author{
J.V. Ramani, Anil K. Karn and Sunil Yadav \\ Agra college and University of Delhi, India
}

ABstRaCt. In this paper we initiate the study of ordered $\mathcal{F}$-bimodules as the inductive limit of matrix ordered spaces.

\section{INTRODUCTION}

Choi and Effros [1] characterized operator systems as a particular type of matrix ordered spaces. Ruan characterizes operator spaces as a particular type of matrix normed spaces. In both these cases we consider a matricial structure on a complex vector space. Pointing out at this structure B.E.Johnson suggested that "the theory of matricially normed spaces might be simplified if one instead considered normed modules over the infinite matrix algebra" [5, section 4]. In [5] Effros and Ruan verified this hypothesis for matrix normed spaces. In this paper we take an initiative to work on this hypothesis in the direction of matrix ordered spaces (c.f. [7, 6]). In section 2 , we recall the characterization of non-degenerate $\mathcal{F}$-bimodules, in terms of inductive limit spaces [5]. We extend the above characterization to a $*$-structure. In section 3 , we describe the inductive limit of matrix ordered spaces in terms of ordered $\mathcal{F}$-bimodules and their related properties.

We begin by recalling some definitions and facts which we need in this paper.

\section{Matricial notions.}

Let $V$ be a complex vector space. Let $M_{n}(V)$ denote the set of all $n \times n$ matrices with entries from $V$. For $V=\mathcal{C}$, we denote $M_{n}(\mathcal{C})$ by $M_{n}$. For

2000 Mathematics Subject Classification. 46L07.

Key words and phrases. $\mathcal{F}$-bimodule, direct limit. 
$\alpha=\left[\alpha_{i j}\right] \in M_{n}$ and $v=\left[v_{i j}\right] \in M_{n}(V)$ we define

$$
\alpha v=\left[\sum_{j=1}^{n} \alpha_{i j} v_{j k}\right], \quad v \alpha=\left[\sum_{j=1}^{n} v_{i j} \alpha_{j k}\right] .
$$

Then $M_{n}(V)$ is a $M_{n}$-bimodule for all $n \in \mathcal{N}$. In particular $M_{n}(V)$ is a complex vector space for all $n \in \mathcal{N}$. For $v \in M_{n}(V), w \in M_{m}(V)$, we define

$$
v \oplus w=\left[\begin{array}{cc}
v & 0 \\
0 & w
\end{array}\right] \in M_{n+m}(V) .
$$

Next, we consider the family $\left\{M_{n}\right\}$. For each $n, m \in \mathcal{N}$ define $\sigma_{n, n+m}$ : $M_{n} \longrightarrow M_{n+m}$ given by $\sigma_{n, n+m}(\alpha)=\alpha \oplus 0_{m}$. Then $\sigma_{n, n+m}$ is a vector space isomorphism with

$$
\sigma_{n, n+m}(\alpha \beta)=\sigma_{n, n+m}(\alpha) \sigma_{n, n+m}(\beta) .
$$

Thus we may "identify" $M_{n}$ in $M_{n+m}$ as a subalgebra for every $m \in \mathcal{N}$. More generally, we may identify $M_{n}$ in the set $\mathcal{F}$ of $\infty \times \infty$ complex matrices, having entries zero after first $n$ rows and first $n$ columns. Then $\mathcal{F}$ may be considered as the direct or inductive limit of the family $\left\{M_{n}\right\}$. In this sense

$$
\mathcal{F}=\bigcup_{n=1}^{\infty} M_{n}
$$

Let $e_{i j}$ denote the $\infty \times \infty$ matrix with 1 at the $(i, j)$ th entry and 0 elsewhere. Then the collection $\left\{e_{i j}\right\}$ is called the set of matrix units in $\mathcal{F}$. We write $1_{n}$ for $\sum_{i=1}^{n} e_{i i}$.

For $i, j, k, l \in \mathcal{N}$, we have $e_{i j} e_{k l}=\delta_{j k} e_{i l}$. Note that for any $\alpha \in \mathcal{F}$, there exist complex numbers $\alpha_{i j}$ such that

$$
\alpha=\sum_{i, j} \alpha_{i j} e_{i j} \quad \text { ( a finite sum). }
$$

Thus $\mathcal{F}$ is an algebra.

For $\alpha=\sum_{i, j} \alpha_{i j} e_{i j} \in \mathcal{F}$, we define $\alpha^{*}=\sum_{i, j} \bar{\alpha}_{j i} e_{i j} \in \mathcal{F}$. Then $\alpha \longmapsto \alpha^{*}$ is an involution. In other words, $\mathcal{F}$ is a $*$-algebra.

\section{Matricial inductive Limit of $*$-VeCtor SPaCES}

Let $V$ be a complex vector space. Consider the family $\left\{M_{n}(V)\right\}$. For each $n, m \in \mathcal{N}$, define $T_{n, n+m}: M_{n}(V) \longrightarrow M_{n+m}(V)$ given by $T_{n, n+m}(v)=$ $v \oplus 0_{m}, 0_{m} \in M_{m}(V)$. Then $T_{n, n+m}$ is an injective homomorphism. Let $\mathcal{V}$ be the inductive limit of the directed family $\left\{M_{n}(V), T_{n, n+m}\right\}$. We shall call $\mathcal{V}$ the matricial inductive limit or direct limit of $V$. The following observations may be obtained from [5]:

1. $\mathcal{V}$ is an $\mathcal{F}$-bimodule.

2. $\mathcal{V} \cong V \otimes \mathcal{F}$. 
Definition 2.1. Any $\mathcal{F}$-bimodule $\mathcal{W}$ is said to be non-degenerate if for every $v \in \mathcal{W}$ there exists a $n \in \mathcal{N}$ such that $1_{n} v 1_{n}=v$.

It follows from [5] that

Proposition 2.2. $\mathcal{V}$ is non-degenerate.

The matricial inductive limit of a complex vector space $V$ may be characterized in the following sense:

Theorem 2.3. Let $\mathcal{W}$ be a non-degenerate $\mathcal{F}$-bimodule. Put $W=$ $e_{11} \mathcal{W} e_{11}$. Then $W$ is a complex vector space and $\mathcal{W}$ is its matricial inductive limit [5].

COROLlary 2.4. $M_{n}(W) \cong 1_{n} \mathcal{W} 1_{n}$ for all $n \in \mathcal{N}$.

REMARK 2.5. Let $V$ be a complex vector space. Let $\mathcal{V}$ be its matricial inductive limit. Then

(a) $\mathcal{V}=\bigcup_{n=1}^{\infty} M_{n}(V) \cong V \otimes \mathcal{F}$.

(b) $M_{n}(V) \cong V \otimes M_{n}$ for all $n \in \mathcal{N}$.

Next we consider the matricial inductive (direct) limit of $*$-vector spaces.

Definition 2.6. Let $\mathcal{W}$ be an $\mathcal{F}$-bimodule. Then a map $*: \mathcal{W} \longrightarrow \mathcal{W}$ is called an involution on $\mathcal{W}$ if

(1) $\left(v^{*}\right)^{*}=v$

(2) $(v+w)^{*}=v^{*}+w^{*}$

(3) $(\alpha v)^{*}=v^{*} \alpha^{*}, \quad(v \alpha)^{*}=\alpha^{*} v^{*}$ for all $v \in \mathcal{W}, \alpha \in \mathcal{F}$.

In this case $\mathcal{W}$ is called $a * \mathcal{F}$-bimodule.

TheOREM 2.7. Let $V$ be $a *$ vector space. Let $\mathcal{V}$ denote the matricial inductive limit of $V$. Then $\mathcal{V}$ is a nondegenerate $*-\mathcal{F}$-bimodule. Conversely, let $\mathcal{W}$ be a nondegenerate $*-\mathcal{F}$-bimodule. Put $W=e_{11} \mathcal{W} e_{11}$. Then $W$ is a* vector space and $\mathcal{W}$ is the matricial inductive limit of $W$.

Proof. First, let $V$ be a $*$-vector space and $\mathcal{V}$ be the matricial inductive limit of $V$. Then by Proposition 2.2, $\mathcal{V}$ is a non-degenerate $\mathcal{F}$-bimodule. We now define a $*$-structure on $\mathcal{V}$. Let $v \in \mathcal{V}$. Then we have $v=\sum_{i, j} v_{i j} \otimes e_{i j}$ for some $v_{i j} \in V$. Define $v^{*}=\sum_{i, j} v_{j i}^{*} \otimes e_{i j}$. Then $v \mapsto v^{*}$ defines an involution on $\mathcal{V}$ so that $\mathcal{V}$ is a nondegenerate $*-\mathcal{F}$-bimodule.

Conversely, let $\mathcal{W}$ be non-degenerate ${ }^{*}-\mathcal{F}$-bimodule. Put $W=1_{n} \mathcal{W} 1_{n}$. Then by Theorem 2.3 , we have $\mathcal{W} \cong W \otimes \mathcal{F}$ and it is routine to verify that $W$ is a $*$-vector space.

Put $\mathcal{W}_{s a}=\left\{w \in \mathcal{W} \mid w=w^{*}\right\}$. Then we have

CoROLlary 2.8. $1_{n} \mathcal{W}_{s a} 1_{n} \cong M_{n}(W)_{\text {sa }}$ for all $n \in \mathcal{N}$.

COROLLARY 2.9. $\mathcal{W}_{s a}=\bigcup_{n=1}^{\infty} M_{n}(W)_{s a}$. 


\section{InduCtive LIMIT OF MATRIX ORDERED SPACES}

Definition 3.1 (Matrix ordered space). A matrix ordered space is a* vector space $V$ together with a cone $M_{n}(V)^{+}$in $M_{n}(V)_{\text {sa }}$ for all $n \in \mathcal{N}$ and with the following property: if $v \in M_{n}(V)^{+}$and $\gamma \in M_{n, m}$ then $\gamma^{*} v \gamma \in$ $M_{m}(V)^{+}$for any $n, m \in \mathcal{N}$.

Definition 3.2 (Ordered $\mathcal{F}$-bimodule). Let $\mathcal{V}$ be a $*$ - $\mathcal{F}$-bimodule. Let $\mathcal{V}^{+}$be a bimodule cone in $\mathcal{V}_{\text {sa }}$. That is

1. $v_{1}, v_{2} \in \mathcal{V}^{+} \Rightarrow v_{1}+v_{2} \in \mathcal{V}^{+}$.

2. $v \in \mathcal{V}^{+}, \alpha \in \mathcal{F} \Rightarrow \alpha^{*} v \alpha \in \mathcal{V}^{+}$.

Then $\left(\mathcal{V}, \mathcal{V}^{+}\right)$will be called an ordered $\mathcal{F}$-bimodule.

REMARK 3.3. Let $\mathcal{V}$ be non-degenerate. Then $\mathcal{V}_{s a}$ and consequently $\mathcal{V}^{+}$ are both nondegenerate.

Theorem 3.4. Let $\left(V,\left\{M_{n}(V)^{+}\right\}\right)$be a matrix ordered space. Let $\mathcal{V}$ be the matricial inductive limit of $V$. Then $\left(\mathcal{V}, \mathcal{V}^{+}\right)$is a non-degenerate ordered $\mathcal{F}$-bimodule, where $\mathcal{V}^{+}=\bigcup_{n=1}^{\infty} M_{n}(V)^{+}$. Conversely, let $\left(\mathcal{W}, \mathcal{W}^{+}\right)$be a nondegenerate ordered $\mathcal{F}$-bimodule. Put $W=1_{1} \mathcal{W} 1_{1}$ and $M_{n}(W)^{+}=1_{n} \mathcal{W}^{+} 1_{n}$ for all $n \in \mathcal{N}$. Then $\left(W,\left\{M_{n}(W)^{+}\right\}\right)$is a matrix ordered space with $\mathcal{W}^{+}=$ $\bigcup_{n=1}^{\infty} M_{n}(W)^{+}$.

Proof. We prove only the non-trivial part. Let $\left(\mathcal{W}, \mathcal{W}^{+}\right)$be a nondegenerate ordered $\mathcal{F}$-bimodule. Then $\mathcal{W}$ is a non-degenerate $* \mathcal{F}$-bimodule. Hence by the Theorem $2.7, W$ is a $*$ vector space and $1_{n} \mathcal{W}_{s a} 1_{n} \cong M_{n}(W)_{s a}$ for all $n \in \mathcal{N}$. Since $\mathcal{W}^{+} \subseteq \mathcal{W}_{\text {sa }}$, therefore $1_{n} \mathcal{W}^{+} 1_{n} \subseteq 1_{n} \mathcal{W}_{\text {sa }} 1_{n}$ for all $n \in \mathcal{N}$. Thus $M_{n}(W)^{+} \subseteq M_{n}(W)_{s a}$ for all $n \in \mathcal{N}$. We show that $M_{n}(W)^{+}$is a cone in $M_{n}(W)_{s a}$ for all $n \in \mathcal{N}$.

(1) Let $u, v \in M_{n}(W)^{+}, n \in \mathcal{N}$. Then $u=1_{n} \bar{u} 1_{n}, v=1_{n} \bar{v} 1_{n}$ for some $\bar{u}, \bar{v} \in \mathcal{W}^{+}$. Then $\bar{u}+\bar{v} \in \mathcal{W}^{+}$as $\mathcal{W}^{+}$is a cone. Thus

$$
u+v=1_{n}(\bar{u}+\bar{v}) 1_{n} \in M_{n}(W)^{+} .
$$

(2) Let $\alpha \geq 0, u \in M_{n}(W)^{+}$. Then $u=1_{n} \bar{u} 1_{n}$ for some $\bar{u} \in \mathcal{W}^{+}$. Put $\alpha_{n}=\sqrt{\alpha} 1_{n}$. Then

$$
\alpha u=\alpha\left(1_{n} \bar{u} 1_{n}\right)=\alpha_{n} \bar{u} \alpha_{n}=1_{n}\left(\alpha_{n} \bar{u} \alpha_{n}\right) 1_{n} \in M_{n}(W)^{+} .
$$

Therefore $M_{n}(W)^{+}$is a cone in $M_{n}(W)_{s a}$ for every $n \in \mathcal{N}$. We now show $\left(W,\left\{M_{n}(W)^{+}\right\}\right)$is a matrix ordered space. Let $u \in M_{n}(W)^{+}$and $\alpha \in M_{n, m}$. Then, $u=1_{n} \bar{u} 1_{n}$ for some $\bar{u} \in \mathcal{W}^{+}$. Note that $1_{n} \alpha=\alpha=\alpha 1_{m}$ so that $\alpha^{*} 1_{n}=\alpha^{*}=1_{m} \alpha^{*}$. Thus

$$
\alpha^{*} u \alpha=\alpha^{*} 1_{n} \bar{u} 1_{n} \alpha=1_{m} \alpha^{*} \bar{u} \alpha 1_{m} \in M_{m}(W)^{+} .
$$

Therefore $\left(W,\left\{M_{n}(W)^{+}\right\}\right)$is a matrix ordered space. By Theorem 2.7 and its Corollary 2.9, $\mathcal{W}=\bigcup_{n=1}^{\infty} M_{n}(W)$ and $\mathcal{W}_{s a}=\bigcup_{n=1}^{\infty} M_{n}(W)_{s a}$. We now 
claim $\mathcal{W}^{+}=\bigcup_{n=1}^{\infty} M_{n}(W)^{+} . M_{n}(W)^{+}=1_{n} \mathcal{W}^{+} 1_{n} \subseteq \mathcal{W}^{+}$for all $n$. Therefore $\bigcup_{n=1}^{\infty} M_{n}(W)^{+} \subseteq \mathcal{W}^{+}$. Let $w \in \mathcal{W}^{+} . \Rightarrow w \in \mathcal{W}_{s a}=\bigcup_{n=1}^{\infty} M_{n}(W)_{s a}$. $\Rightarrow w \in M_{p}(W)_{s a}$ for some $p \in \mathcal{N}$. Then $1_{p} w 1_{p}=w$. Also by definition $1_{p} w 1_{p} \in M_{p}(W)^{+}$. Therefore $w \in M_{p}(W)^{+} \subseteq \bigcup_{n=1}^{\infty} M_{n}(W)^{+}$. Hence $\mathcal{W}^{+}=\bigcup_{n=1}^{\infty} M_{n}(W)^{+}$.

Definition 3.5. Let $\left(V,\left\{M_{n}(V)^{+}\right\}\right)$be a matrix ordered space. Let $\mathcal{V}$ be the matricial inductive limit of $V$. Set $\mathcal{V}^{+}=\bigcup_{n=1}^{\infty} M_{n}(V)^{+}$. Then the ordered $\mathcal{F}$-bimodule $\left(\mathcal{V}, \mathcal{V}^{+}\right)$is called the (matricial) inductive limit or direct limit of the matrix ordered space $\left(V,\left\{M_{n}(V)^{+}\right\}\right)$.

We recall the following from [1]:

Definition 3.6. Let $\left(V,\left\{M_{n}(V)^{+}\right\}\right)$be a matrix ordered space. We say that $V^{+}$is proper if $V^{+} \cap\left(-V^{+}\right)=\{0\}$.

It is shown in [1] that if $V^{+}$is proper, then so is $M_{n}(V)^{+}$for all $n$. We extend this idea to ordered $\mathcal{F}$-bimodules.

Definition 3.7. Let $\left(\mathcal{V}, \mathcal{V}^{+}\right)$be an ordered $\mathcal{F}$-bimodule. We say $\mathcal{V}^{+}$is proper if $\mathcal{V} \cap\left(-\mathcal{V}^{+}\right)=\{0\}$.

REMARK 3.8. If $\left(\mathcal{V}, \mathcal{V}^{+}\right)$is the direct limit of $\left(V,\left\{M_{n}(V)^{+}\right\}\right)$and if $\mathcal{V}^{+}$ is proper then $M_{n}(V)^{+}$is proper for each $n \in \mathcal{N}$. In fact if $v \in M_{n}(V)^{+} \cap$ $\left(-M_{n}(V)^{+}\right)$, then $v \in \mathcal{V}^{+} \cap\left(-\mathcal{V}^{+}\right)=\{0\}$, implies $v=0$.

Hence $M_{n}(V)^{+}$is proper for each $n \in \mathcal{N}$.

Theorem 3.9. Let $\left(\mathcal{V}, \mathcal{V}^{+}\right)$be the direct limit of $\left(V,\left\{M_{n}(V)^{+}\right\}\right)$. Then $V^{+}$is proper if and only if $\mathcal{V}^{+}$is proper.

Proof. Let $\mathcal{V}^{+}$be proper. The above remark gives that $M_{n}(V)^{+}$is proper for each $n \in \mathcal{N}$. In particular, $V^{+}$is proper.

Conversely, let $V^{+}$be proper. Let $v \in \mathcal{V}^{+} \cap\left(-\mathcal{V}^{+}\right)$or $\pm v \in \mathcal{V}^{+}$. Then

$$
\pm e_{1 i} v e_{i 1} \in e_{1 i} \mathcal{V}^{+} e_{i 1} \text { for all } i \in \mathcal{N}
$$

We show $e_{1 i} \mathcal{V}^{+} e_{i 1} \subseteq V^{+}$. By definition $V^{+}=e_{11} \mathcal{V}^{+} e_{11}$. Also $e_{11} e_{1 i}=$ $e_{1 i}, e_{i 1} e_{11}=e_{i 1}, e_{1 i}^{*}=e_{i 1}$ so that $e_{1 i} \mathcal{V}^{+} e_{i 1} \subseteq \mathcal{V}^{+}$, for $\mathcal{V}^{+}$is a bimodule cone. It follows that

$$
e_{11} e_{1 i} \mathcal{V}^{+} e_{i 1} e_{11} \subseteq e_{11} \mathcal{V}^{+} e_{11}=V^{+} .
$$

In other words $e_{1 i} \mathcal{V}^{+} e_{i 1} \subseteq V^{+}$for all $i \in \mathcal{N}$. From $(I I I)$, we have $\pm e_{1 i} v e_{i 1} \in$ $V^{+}$for all $i \in \mathcal{N}$. Since $V^{+}$is proper therefore $e_{1 i} v e_{i 1}=0$ for all $i \in \mathcal{N}$. Put $e_{1 i} v e_{j 1}=v_{i j}$ for all $i, j \in \mathcal{N}$. Then $v_{i i}=0$ for all $i \in \mathcal{N}$. Since $v \in \mathcal{V}^{+} \subseteq \mathcal{V}_{s a}$, we have

$$
v_{i j}^{*}=e_{1 j} v^{*} e_{i 1}=e_{1 j} v e_{i 1}=v_{j i}
$$

for all $i, j \in \mathcal{N}$. Let $i \neq j$. Then by a similar argument,

$$
\left(e_{1 i}+e_{1 j}\right)( \pm v)\left(e_{i 1}+e_{j 1}\right) \in V^{+} .
$$


Also $\left(e_{i 1}+e_{j 1}\right)^{*}=e_{i 1}^{*}+e_{j 1}^{*}=e_{1 i}+e_{1 j}$. Therefore

$$
e_{11}\left[\left(e_{1 i}+e_{1 j}\right)( \pm v)\left(e_{i 1}+e_{j 1}\right)\right] e_{11} \in e_{11} \mathcal{V}^{+} e_{11}=V^{+} .
$$

This implies that

$$
\pm\left[e_{1 i} v e_{i 1}+e_{1 i} v e_{j 1}+e_{1 j} v e_{i 1}+e_{1 j} v e_{j 1}\right] \in \mathcal{V}^{+} .
$$

Thus $\pm\left[v_{i j}+v_{j i}\right] \in V^{+}$. Since $V^{+}$is proper, we get $v_{i j}+v_{j i}=0$. Similarly, by considering

$$
\left(e_{1 i}-e_{1 j}\right)( \pm v)\left(e_{i 1}-e_{j 1}\right) \in V^{+}
$$

we get $v_{i j}-v_{j i}=0$. Therefore $v_{i j}=0$ for all $i, j \in \mathcal{N}$. Now for a $v \in \mathcal{V}$ there exists a $n \in \mathcal{N}$ such that $1_{n} v 1_{n}=v$. It follows that

$$
\begin{aligned}
v & =\left(\sum_{i=1}^{n} e_{i i}\right) v\left(\sum_{j=1}^{n} e_{j j}\right) \\
& =\left(\sum_{i=1}^{n} e_{i 1} e_{1 i}\right) v\left(\sum_{j=1}^{n} e_{j 1} e_{1 j}\right) \\
& =\sum_{i, j=1}^{n} e_{i 1}\left(e_{1 i} v e_{j 1}\right) e_{1 j} \\
& =\sum_{i, j=1}^{n} e_{i 1} v_{i j} e_{1 j}=0 .
\end{aligned}
$$

Hence $\mathcal{V}^{+}$is proper.

Now we consider another notion related to order theory.

Definition 3.10. We say $V^{+}$is generating if given $v \in V$ there are $v_{0}, v_{1}, v_{2}, v_{3} \in V^{+}$such that $v=\sum_{k=0}^{3} i^{k} v_{k}$, where $i^{2}=-1$.

Definition 3.11. Let $\left(\mathcal{V}, \mathcal{V}^{+}\right)$be an ordered $\mathcal{F}$-bimodule. Then we say $\mathcal{V}^{+}$is generating if given $v \in \mathcal{V}$ there exist $v_{0}, v_{1}, v_{2}, v_{3} \in \mathcal{V}^{+}$such that $v=$ $\sum_{k=0}^{3} i^{k} v_{k}$, where $i^{2}=-1$.

Theorem 3.12. Let $\left(\mathcal{V}, \mathcal{V}^{+}\right)$be the direct limit of $\left(V,\left\{M_{n}(V)^{+}\right\}\right)$. Then $V^{+}$is generating if and only if $\mathcal{V}^{+}$is generating.

Proof. First, let $\mathcal{V}^{+}$be generating. Let $v \in V$. Then $v=1_{1} \bar{v} 1_{1}$ for some $\bar{v} \in \mathcal{V}$. Therefore there exists $\bar{v}_{0}, \bar{v}_{1}, \bar{v}_{2}, \bar{v}_{3} \in \mathcal{V}^{+}$such that $\bar{v}=\sum_{k=0}^{3} i^{k} \bar{v}_{k}$. Now $1_{1} \bar{v} 1_{1}=\sum_{k=0}^{3} i^{k} 1_{1} \bar{v}_{k} 1_{1}$ and Since $V^{+}=1_{1} \mathcal{V}^{+} 1_{1}$ so that

$$
v=1_{1} \bar{v} 1_{1}=\sum_{k=0}^{3} i^{k} 1_{1} \bar{v}_{k} 1_{1}=\sum_{k=0}^{3} i^{k} w_{k}, \quad w_{0}, w_{1}, w_{2}, w_{3} \in V^{+}
$$

where $w_{k}=1_{1} \bar{v}_{k} 1_{1}$. Therefore $V^{+}$is generating. 
Conversely, let $V^{+}$be generating. We show that $\mathcal{V}^{+}$is generating. Let $v \in \mathcal{V}$. Then there exists a $n \in \mathcal{N}$ such that $1_{n} v 1_{n}=v$. Put $e_{1 r} v e_{s 1}=v_{r s}$. Then

$$
v=\sum_{r, s=1}^{n} e_{r 1} v_{r s} e_{1 s}, \quad v_{r s} \in V .
$$

Since $V^{+}$is generating there exist $v_{r s}^{0}, v_{r s}^{1}, v_{r s}^{2}, v_{r s}^{3} \in V^{+}$such that $v_{r s}=$ $\sum_{k=0}^{3} i^{k} v_{r s}^{k}$. For every $r, s ; \quad 1 \leq r, s \leq n$, put

$$
\begin{aligned}
& u_{r s}^{1}=v_{r s}^{0}+v_{r s}^{2}, \\
& u_{r s}^{2}=v_{r s}^{1}+v_{r s}^{3} .
\end{aligned}
$$

Then $u_{r s}^{1}, u_{r s}^{2} \in V^{+}$for every $r, s=1, \ldots, n$. Next put

$$
u_{r s}=u_{r s}^{1}+u_{r s}^{2} \text {, }
$$

for all $r, s, 1 \leq r, s \leq n$, and let $u_{1}=\sum_{r, s=1}^{n} e_{r 1} u_{r s} e_{1 r}$ and $u_{2}=$ $\sum_{r, s=1}^{n} e_{s 1} u_{r s} e_{1 s}$. Then $u_{1}, u_{2} \in \mathcal{V}^{+}$. Further put

$$
\begin{aligned}
v_{0} & =\frac{1}{4}\left(u_{1}+u_{2}+v+v^{*}\right) \\
v_{1} & =\frac{1}{4}\left(u_{1}+u_{2}-i v+i v^{*}\right) \\
v_{2} & =\frac{1}{4}\left(u_{1}+u_{2}-v-v^{*}\right) \\
v_{3} & =\frac{1}{4}\left(u_{1}+u_{2}+i v-i v^{*}\right) .
\end{aligned}
$$

Then we have $v=\sum_{k=0}^{3} i^{k} v_{k}$. We will be done if we can show that $v_{k} \in$ $\mathcal{V}^{+}, k=0,1,2,3$. Since Rev $v_{r s}=v_{r s}^{0}-v_{r s}^{2}$ and $\operatorname{Im} v_{r s}=v_{r s}^{1}-v_{r s}^{3}, 1 \leq r, s \leq n$, we have $u_{r s}^{1} \pm R e v_{r s} \in V^{+}$and $u_{r s}^{2} \pm I m v_{r s} \in V^{+}$for all $r, s=1, . ., n$. Thus

$$
\begin{aligned}
b= & \frac{1}{2}\left(e_{11}+e_{21}\right)\left(u_{r s}^{1}+\operatorname{Rev}_{r s}\right)\left(e_{11}+e_{12}\right) \\
& +\frac{1}{2}\left(e_{11}-e_{21}\right)\left(u_{r s}^{1}-\operatorname{Rev}_{r s}\right)\left(e_{11}-e_{12}\right) \in \mathcal{V}^{+}
\end{aligned}
$$

and

$$
\begin{aligned}
c= & \frac{1}{2}\left(e_{11}-i e_{21}\right)\left(u_{r s}^{2}+\operatorname{Im}_{r s}\right)\left(e_{11}+i e_{12}\right) \\
& +\frac{1}{2}\left(e_{11}+i e_{21}\right)\left(u_{r s}^{2}-\operatorname{Im} v_{r s}\right)\left(e_{11}-i e_{12}\right) \in \mathcal{V}^{+} .
\end{aligned}
$$

It follows that

$$
a=\sum_{r, s=1}^{n}\left(e_{r 1}+e_{n+s, 2}\right)(b+c)\left(e_{1 r}+e_{2, n+s}\right) \in M_{2 n}(V)^{+} \subset \mathcal{V}^{+}
$$


Now

$$
\begin{aligned}
& a=\frac{1}{2}\left[\sum _ { r , s = 1 } ^ { n } ( e _ { r 1 } + e _ { n + s , 2 } ) \left[\left(e_{11}+e_{21}\right)\left(u_{r s}^{1}+R e v_{r s}\right)\left(e_{11}+e_{12}\right)\right.\right. \\
& +\left(e_{11}-e_{21}\right)\left(u_{r s}^{1}-R e v_{r s}\right)\left(e_{11}-e_{12}\right) \\
& +\left(e_{11}-i e_{21}\right)\left(u_{r s}^{2}+I m v_{r s}\right)\left(e_{11}+i e_{12}\right) \\
& \left.\left.+\left(e_{11}+i e_{21}\right)\left(u_{r s}^{2}-I m v_{r s}\right)\left(e_{11}-i e_{12}\right)\right]\left(e_{1 r}+e_{2, n+s}\right)\right] \\
& =\frac{1}{2}\left[\sum _ { r , s = 1 } ^ { n } \left[\left(e_{r 1}+e_{n+s, 1}\right)\left(u_{r s}^{1}+\operatorname{Rev}_{r s}\right)\left(e_{1 r}+e_{1, n+s}\right)\right.\right. \\
& +\left(e_{r 1}-e_{n+s, 1}\right)\left(u_{r s}^{1}-\operatorname{Rev}_{r s}\right)\left(e_{1 r}-e_{1, n+s}\right) \\
& +\left(e_{r 1}-i e_{n+s, 1}\right)\left(u_{r s}^{2}+I m v_{r s}\right)\left(e_{1 r}+i e_{1, n+s}\right) \\
& \left.\left.+\left(e_{r 1}+i e_{n+s, 1}\right)\left(u_{r s}^{2}-\operatorname{Imv}_{r s}\right)\left(e_{1 r}-i e_{1, n+s}\right)\right]\right] \\
& =\sum_{r, s=1}^{n} e_{r 1}\left(v_{r s}^{0}+v_{r s}^{1}+v_{r s}^{2}+v_{r s}^{3}\right) e_{1 r} \\
& +\sum_{r, s=1}^{n} e_{n+s, 1}\left(v_{r s}^{0}+v_{r s}^{1}+v_{r s}^{2}+v_{r s}^{3}\right) e_{1, n+s} \\
& +\sum_{r, s=1}^{n} e_{r 1}\left(v_{r s}^{0}-v_{r s}^{2}+i\left(v_{r s}^{1}-v_{r s}^{3}\right)\right) e_{1, n+s} \\
& +\sum_{r, s=1}^{n} e_{n+s, 1}\left(v_{r s}^{0}-v_{r s}^{2}-i\left(v_{r s}^{1}-v_{r s}^{3}\right)\right) e_{1 r} \\
& =\sum_{r, s=1}^{n} e_{r 1} u_{r s} e_{1 r}+\sum_{r, s=1}^{n} e_{n+s, 1} u_{r s} e_{1, n+s} \\
& +\sum_{r, s=1}^{n} e_{r 1} v_{r s} e_{1, n+s}+\sum_{r, s=1}^{n} e_{n+s, 1} v_{r s}^{*} e_{1 r} .
\end{aligned}
$$

Put

$$
\begin{gathered}
\alpha=\sum_{r=1}^{n} e_{r r}+\sum_{s=1}^{n} e_{n+s, s}, \quad \beta=\sum_{r=1}^{n} e_{r r}-\sum_{s=1}^{n} e_{n+s, s}, \\
\gamma=\sum_{r=1}^{n} e_{r r}-i \sum_{s=1}^{n} e_{n+s, s}, \quad \delta=\sum_{r=1}^{n} e_{r r}+i \sum_{s=1}^{n} e_{n+s, s} .
\end{gathered}
$$

We claim that

$$
\begin{array}{rlrl}
v_{0} & =\frac{1}{4}\left(\alpha^{*} a \alpha\right), & v_{1} & =\frac{1}{4}\left(\gamma^{*} a \gamma\right) \\
v_{2}=\frac{1}{4}\left(\beta^{*} a \beta\right), & v_{3}=\frac{1}{4}\left(\delta^{*} a \delta\right) .
\end{array}
$$


We verify only that $v_{0}=\frac{1}{4}\left(\alpha^{*} a \alpha\right)$ and others follow similarly. Now using (II), we get

$$
\begin{gathered}
\alpha^{*} a \alpha=\left(\sum_{k=1}^{n} e_{k k}+\sum_{l=1}^{n} e_{l, n+l}\right)\left(\sum_{r, s=1}^{n} e_{r 1} u_{r s} e_{1 r}+e_{n+s, 1} u_{r s} e_{1, n+s}\right. \\
\left.+e_{r 1} v_{r s} e_{1, n+s}+e_{n+s, 1} v_{r s}^{*} e_{1 r}\right)\left(\sum_{p=1}^{n} e_{p p}+\sum_{q=1}^{n} e_{n+q, q}\right) \\
=\sum_{r, s, k, p=1}^{n}\left[e_{k k}\left(e_{r 1} u_{r s} e_{1 r}\right) e_{p p}+e_{k k}\left(e_{n+s, 1} u_{r s} e_{1, n+s}\right) e_{p p}\right. \\
\left.+e_{k k}\left(e_{r 1} v_{r s} e_{1, n+s}\right) e_{p p}+e_{k k}\left(e_{n+s, 1} v_{r s}^{*} e_{1 r}\right) e_{p p}\right] \\
+\sum_{r, s, k, q=1}^{n}\left[e_{k k}\left(e_{r 1} u_{r s} e_{1 r}\right) e_{n+q, q}+e_{k k}\left(e_{n+s, 1} u_{r s} e_{1, n+s}\right) e_{n+q, q}\right. \\
\left.+e_{k k}\left(e_{r 1} v_{r s} e_{1, n+s}\right) e_{n+q, q}+e_{k k}\left(e_{n+s, 1} v_{r s}^{*} e_{1 r}\right) e_{n+q, q}\right] \\
+\sum_{r, s, l, p=1}^{n}\left[e_{l, n+l}\left(e_{r 1} u_{r s} e_{1 r}\right) e_{p p}+e_{l, n+l}\left(e_{n+s, 1} u_{r s} e_{1, n+s}\right) e_{p p}\right. \\
\left.+e_{l, n+l}\left(e_{r 1} v_{r s} e_{1, n+s}\right) e_{p p}+e_{l, n+l}\left(e_{n+s, 1} v_{r s}^{*} e_{1 r}\right) e_{p p}\right] \\
+\sum_{r, s, l, q=1}^{n}\left[e_{l, n+l}\left(e_{r 1} u_{r s} e_{1 r}\right) e_{n+q, q}+e_{l, n+l}\left(e_{n+s, 1} u_{r s} e_{1, n+s}\right) e_{n+q, q}\right. \\
\left.+e_{l, n+l}\left(e_{r 1} v_{r s} e_{1, n+s}\right) e_{n+q, q}+e_{l, n+l}\left(e_{n+s, 1} v_{r s}^{*} e_{1 r}\right) e_{n+q, q}\right] \\
+\sum_{r, s, k, p=1}^{n} \delta_{k r} \delta_{r p} e_{k 1} u_{r s} e_{1 p}+\sum_{r, s, k, q=1}^{n} \delta_{k r} \delta_{s q} e_{k 1} v_{r s} e_{1 q} \\
+\sum_{r, p=1}^{n} \delta_{l s} \delta_{r p} e_{l 1} v_{r s}^{*} e_{1 p}+\sum_{r, s, l, q=1}^{n} \delta_{l s} \delta_{s q} e_{l 1} u_{r s} e_{1 q}
\end{gathered}
$$

That is

$$
\begin{aligned}
&\left(\alpha^{*} a \alpha\right)= \sum_{r, s=1}^{n} e_{r 1} u_{r s} e_{1 r}+\sum_{r, s=1}^{n} e_{r 1} v_{r s} e_{1 s} \\
& \quad+\sum_{r, s=1}^{n} e_{s 1} v_{r s}^{*} e_{1 r}+\sum_{r, s=1}^{n} e_{s 1} u_{r s} e_{1 s} \\
&=u_{1}+v+v^{*}+u_{2} .
\end{aligned}
$$

Hence our claim is proved. Therefore $\mathcal{V}^{+}$is generating.

ACKNowledgements.

The authors are greatful to the referees for their valuable suggestions. 


\section{REFERENCES}

[1] M.D. Choi and E.G. Effros, Injectivity and operator spaces, J. Funct. Anal., 24 (1977), 156-209.

[2] G.J.O. Jameson, Ordered linear spaces, Lecture Notes in mathematics 104, SpringerVerlag, Berlin, 1972.

[3] Y.C. Wong and K.F. Ng, Partially ordered topological vector spaces, Oxford Mathematical Monograph, Clarendon Press, Oxford, 1973.

[4] Z.J. Ruan, Subspaces of $C^{*}$-algebras, J. Funct. Anal., 76 (1988) 217-230.

[5] E.G. Effros and Z.J. Ruan, On matricially normed spaces, Pacific J. Math., 132 (1988), 243-264.

[6] A.K. Karn, Approximate Matrix Order Unit Spaces, University of Delhi, Delhi, 1997, Ph.D Thesis.

[7] A.K. Karn and R. Vasudevan, Approximate matrix order unit spaces, Yokohama Math. J., 44(1997) 73-91.

J.V. Ramani

Department of mathematics

Agra college, Agra

India

E-mail: ramaniji@yahoo.com

A.K. Karn

Department of mathematics

Deen Dayal Upadhyaya College

University of Delhi

Karam Pura, New Delhi 110015

India

E-mail: anilkarn@rediffmail.com

S. Yadav

Department of mathematics

Agra college, Agra

India

E-mail: drsy@rediffmail.com

Received: 9.3.2005.

Revised: 7.4.2005. 